\title{
Economic Fluctuations and Statistical Physics: The Puzzle of Large Fluctuations
}

\author{
H. EUGENE STANLEY*, XAVIER GABAIX, PARAMESWARAN GOPIKRISHNAN, \\ and VASILIKI PLEROU \\ Center for Polymer Studies and Department of Physics, Boston University, 590 Commonwealth Avenue, Boston, \\ MA 02215 U.S.A.; *Author for correspondence (e-mail: hes@bu.edu; fax: 617-353-3783)
}

(Received: 30 July 2004; accepted: 10 March 2005)

\begin{abstract}
We present an overview of recent research applying ideas of statistical physics to try to better understand puzzles regarding economic fluctuations. One of these puzzles is how to describe outliers, phenomena that lie outside of patterns of statistical regularity. We review evidence consistent with the possibility that such outliers may not exist. This possibility is supported by recent analysis of a database containing the bid, ask, and sale price of each trade of every stock. Further, the data support the picture of economic fluctuations, due to Plerou et al., in which a financial market alternates between being in an "equilibrium phase" where market behavior is split roughly equally between buying and selling, and an "out-of-equilibrium phase" where the market is mainly either buying or selling.
\end{abstract}

Key words: Econophysics, power-law distributions, phase transitions, earthquakes

\section{Introduction}

Interactions between economists and physicists have begun to make progress in answering significant questions. In particular, these collaborations have the potential to change the paradigm for understanding economic fluctuations. Until relatively recently, theories of economic fluctuations invoked the label of "outlier" (bubbles and crashes) to describe fluctuations that do not agree with existing theory. These outliers are of interest, as they correspond to extremely large and unpredictable changes of sufficient magnitude to wreak havoc.

The paradigm of "statistical regularity plus outliers" does not exist in the physical sciences. Indeed, if events occur that do not conform to predictions of the appropriate theory, then that theory is immediately relegated to the dustbin and new theories are sought. An example are the "outliers" that led to the demise of classical mechanics, eventually replaced by the theory of relativity.

Traditional economic theory does not predict outliers, but recent analysis of truly huge quantities of empirical data suggests that classic theories not only fail for a few outliers, but that there occur similar outliers of every possible size. In fact, if one analyzes only a small data set (say $10^{4}$ data points), then outliers appear to occur as "rare events." However, when orders of magnitude more data ( $10^{8}$ data points) are analyzed, one finds orders of magnitude more outliers - so ignoring them is not a responsible option, and studying their properties becomes a realistic goal. One finds that the statistical properties of these "outliers" are identical to the statistical properties of everyday fluctuations. For example, a histogram giving the number of fluctuations of a given magnitude $x$ for fluctuations ranging in magnitude from everyday fluctuations to extremely rare fluctuations (financial earthquakes) that occur with a probability of only $10^{8}$ is a perfect straight line in a double-log plot. 


\section{H. E. Stanley et al.}

An analogy with earthquake research is perhaps not entirely inappropriate. If one studies limited data sets, a paradigm arises in which there are everyday (unnoticeable except by sensitive seismometer) "tremors," punctuated from time to time by rare events (earthquakes). Thanks to the empirical work, we now know that the partition of shocks into "tremors" and "earthquakes" is not valid. Rather, if one examines enough data, one sees that the shocks occur for all possible magnitudes. The law named after Gutenberg and Richter refers to a statistical formula that gives all the data from the smallest tremors to the "big ones." This law is that the histogram giving the number of shocks of a given size is a straight line in a log-log plot [1-3] - there are no outliers.

Thus, an inappropriate paradigm can arise when a limited quantity of data are considered in which data are partitioned into everyday events (often describable by one statistical law) and rare events which, since they are not described by the law are terms outliers. Has an inappropriate paradigm arisen in economic research? In economic research, there are fluctuations in stock prices, number of shares trading hands, and total number of fluctuations. Recent empirical studies calculating histograms for all three quantities are linear on log-log plots (albeit with different slopes). In mathematical language, the occurrence probability of such quantity's fluctuations appear to be described by a power law.

In economics, neither the existence of power laws nor the exact exponents has any accepted theoretical basis. Professionally, empirical laws such as the aforementioned power laws are called "stylized facts," a term that to my ear always sounds dismissive. Accordingly, some theoretical understanding is urgently needed or else these laws will continue to be largely irrelevant. Of course facts, even facts without any interpretation, may have practical value. For example, the Gutenberg-Richter law enables one to calculate the risk of a shock (tremor or earthquake) of a given magnitude, and hence informs the building codes of Los Angeles and Tokyo. Similarly, the empirical laws governing economic fluctuations enable one to calculate the risk of an economic shock of a given magnitude.

The lack of a coherent theory is unfortunate, especially in economics where facts without theoretical foundation is considered a deplorable situation. Accordingly, my collaborators and I have been seeking to develop a theoretical framework within which to interpret these new empirical facts, and recently some progress is beginning to occur $[4,5]$. This work is potentially significant since it provides a theoretical framework within which to interpret the new empirical laws. Specifically, the model fulfills these requirements for such a basic "microscopic" model of the stock market. It is founded on realistic features of the stock market, and reflects the view that market participants have of the functioning of the market, as well as the main determinants of their trading behavior.

\section{First Discovery of Scaling and Universality}

That at least some economic phenomena are described by power law tails has been recognized for over 100 years since Pareto investigated the statistical character of the wealth of individuals by modeling them using the scale-invariant distribution

$$
f(x) \sim x^{-\alpha},
$$

where $f(x)$ denotes the number of people having income $x$ or greater than $x$, and $\alpha$ is an exponent that Pareto estimated to be $1.5[6,7]$. Pareto noticed that his result was universal in the sense that it applied to nations "as different as those of England, of Ireland, of Germany, of the Italian cities, and even of Peru." A physicist would say that the universality class of the scaling law (1) includes all the 
aforementioned countries as well as Italian cities, since by definition two systems belong to the same universality class if they are characterized by the same exponents.

In the century following Pareto's discovery, the twin concepts of scaling and universality have proved to be important in a number of scientific fields [8-10]. A striking example was the elucidation of the puzzling behavior of systems near their critical points. Over the past few decades it has come to be appreciated that the scale-free nature of fluctuations near critical points also characterizes a huge number of diverse systems also characterized by strong fluctuations. This set of systems includes examples that at first sight are as far removed from physics as is economics. For example, consider the percolation problem, which in its simplest form consists of placing pixels on a fraction $p$ of randomly-chosen plaquettes of a computer screen. A remarkable fact is that the largest connected component of pixels magically spans the screen at a threshold value $p_{c}$. This purely geometrical problem has nothing to do, at first sight, with critical point phenomena. Nonetheless, the fluctuations that occur near $p=p_{c}$ are scale free and functions describing various aspects of the incipient spanning cluster that appears at $p=p_{c}$ are described by power laws characterized by exponent values that are universal in the sense that they are independent of the details of the computer screen's lattice (square, triangle, and honeycomb). Nowadays, the concepts of scaling and universality provide the conceptual framework for understanding the geometric problem of percolation.

It is becoming clear that almost any system comprised of a large number of interacting units has the potential of displaying power law behavior. Since economic systems are in fact comprised of a large number of interacting units has the potential of displaying power law behavior, it is perhaps not unreasonable to examine economic phenomena within the conceptual framework of scaling and universality [8-19]. We will discuss this topic in detail below.

\section{Inverse Cubic Law of Stock and Commodity Price Fluctuations}

So having embarked on a path guided by these two theoretical concepts, what does one do? Initially, critical phenomena research - guided by the Pareto principles of scaling and universality - was focused finding which systems display scaling phenomena, and on discovering the actual values of the relevant exponents. This initial empirical phase of critical phenomena research proved vital, for only by carefully obtaining empirical values of exponents such as $\alpha$ could scientists learn which systems have the same exponents (and hence belong to the same universality class). The fashion in which physical systems partition into disjoint universality classes proved essential to later theoretical developments such as the renormalization group [10] - which offered some insight into the reasons why scaling and universality seem to hold; ultimately it led to a better understanding of the critical point.

Similarly, our group's initial research in economics - guided by the Pareto principles - has largely been concerned with establishing which systems display scaling phenomena, and with measuring the numerical values of the exponents with sufficient accuracy that one can begin to identify universality classes if they exist. Economics systems differ from often-studied physical systems in that the number of subunits are considerably smaller in contrast to macroscopic samples in physical systems that contain a huge number of interacting subunits, as many as Avogadro's number $6 \times 10^{23}$. In contrast, in an economic system, one initial work was limited to analyzing time series comprising of order of magnitude $10^{3}$ terms, and nowadays with high frequency data the standard, one may have $10^{8}$ terms. Scaling laws of the form of (1) are found that hold over a range of a factor of $\sim 10^{6}$ on the $x$-axis [20-24]. Moreover, these scaling laws appear to be universal in that they, like the Pareto scaling law, hold for different countries [25], for other social organizations [26-28], and even for bird populations [29]. 


\section{H. E. Stanley et al.}

Recent attempts to make models that reproduce the empirical scaling relationships suggest that significant progress on understanding firm growth may be well underway [30-33], leading to the hope of ultimately developing a clear and coherent "theory of the firm." One utility of the recent empirical work is that now any acceptable theory must respect the fact that power laws hold over typically six orders of magnitude; as Axtell put the matter rather graphically: "the power law distribution is an unambiguous target that any empirically accurate theory of the firm must hit" [20].

With this background on power laws and scale invariance in geometry and in economics, we turn now to the well-studied problem of finance fluctuations, where a consistent set of empirical facts is beginning to emerge. One fact that has been confirmed by numerous, mostly independent, studies is that stock price fluctuations are characterized by a scale-invariant cumulative distribution function of the power law form (1) with $\alpha \approx 3$ [34-36]. This result is also universal, in the sense that this inverse cubic law exponent is within the error bars of results for different segments of the economy, different time periods, and different countries - and is the same for stock averages as different as the S\&P and the Hang Seng [37].

This "inverse cubic law" disagrees with the classic work of Ref. [8] on price fluctuations of cotton, which appear to have display scale free behavior (no outliers) but with much fatter tails characterized by $\alpha \approx 1.7$; this work is of interest because if $\alpha<2$, then the distribution is of the Lévy form. To understand this discrepancy, Matia and collaborators have wondered if the reason for the fatter tails of cotton is that cotton is a commodity, and commodities exist in limited supply so that when a commodity is needed one must sometimes pay exhorbitant prices (e.g., electricity in California). Accordingly, they analyzed a large number of commodities, but they found that these commodities have tails described not by $\alpha<2$ but rather by $\alpha \approx 3[38,39]$. Another possible reason is that Mandelbrot analyzed three data sets, each containing only about 2000 points, while the results on stocks typically contain about 40,000 points per stock (and 1000 stocks, or 40,000,000 total data points). This possibility was tested by choosing randomly 2000 points to analyze, but again one cannot obtain $\alpha<2$. A third possible explanation of this discrepancy is that the cotton market was "out of equilibrium," and that such out-of-equilibrium markets have fatter tails - a possibility consistent with recent analysis of stock price fluctuations [40,41]. A fourth possible explanation is that at the time period in which the cotton data were collected, commodities were intrinsically different than they are today when the Matia data were collected, as today commodities are traded in ways not entirely dissimilar to the way that stocks are traded. Still another possibility is that the cotton distribution has $\alpha<2$ in the central region analyzed in 1963, but ultimately crosses over to power law in the distant tails (not analyzed in 1963). This disagreement led to the development of a class of mathematical processes called truncated Lévy distributions - which has attracted the attention of a number of mathematicians and is actually taught in Columbia University's graduate school of finance [42-49]. In any case, one of the challenges of econophysics is to resolve current results with the classic 1963 analysis of Mandelbrot.

Newcomers to the field of scale invariance often ask why a power law does not extend "forever" as it would for a mathematical power law of the form $f(x)=x^{-\alpha}$. This legitimate concern is put to rest by by reflecting on the fact that power laws for natural phenomena are not equalities, but rather are asymptotic relations of the form $f(x) \sim x^{-\alpha}$. Here the tilde denotes asymptotic equality. Thus $f(x)$ is not "approximately equal to" a power law so the notation $f(x) \approx x^{-\alpha}$ is inappropriate. Similarly, $f(x)$ is not proportional to a power law, so the notation $f(x) \propto x^{-\alpha}$ is also inappropriate. Rather, asymptotic equality means that $f(x)$ becomes increasingly like a power law as $x \longrightarrow \infty$. Moreover, crossovers abound in financial data, such as the crossover from power law behavior to simple Gaussian behavior as the time horizon $\triangle$ over which fluctuations are calculated increases beyond about a year (i.e., the power law behavior holds for time horizons up to a month or even a year, but for horizons exceeding a 
year there is a distinct crossover to Guassian behavior. Such crossovers are characteristic also of other scale-free phenomena in the physical sciences $[9,10]$, where the Yule distribution often proves quite useful.

For reasons of this sort, standard statistical fits to data are inappropriate, and often give distinctly erroneous values of the exponent $\alpha$. Rather, one reliable way of estimating the exponent $\alpha$ is to form successive slopes of pairs of points on a log-log plot, since these successive slopes will be monotonic and converge to the true asymptotic exponent $\alpha$. One finds that successive slopes for the empirical data converge rapidly to a value $\alpha \approx 3$ while successive slopes for the model diverge. While it is clear that a simple three-factor model [50] cannot generate power law behavior, it is less clear why the empirical data analyzed appear at first glance to be well approximated by the model. The first fact is that the region of linearity of the data is not so large as in typical modern studies because the total quantity of data analyzed is not that large, since only a low-frequency time series comprising daily data is used. Only 28,094 records are analyzed [50] (not $4 \times 10^{7}$ as in recent studies [36,37]) and the model simulations are presented for limited sample size. The second fact is that when one superposes a curved line (the model) on a straight line (the data), the untrained eye is easily tempted to find agreement where none exists - and closer inspection of Figures $2-5$ of Ref. [50] reveals actually a rather poor agreement between model and data due to the pronounced downward curvature of the model's predictions [51].

\section{Other Scale-Invariant Quantities Describing Economic Fluctuations}

Other quantities characterizing stock movements (such as the volatility, share volume traded, and number of trades) also display a range of power law behavior over a range of typically $\sim 10^{2}$ [52-55]. The exponents characterizing the power law decays are different for different quantities; it is tempting to conjecture that in finance there may exist a set of relations among the power law exponents found, just as there exist relations among the exponents characterizing different quantities near the critical point. Finally, it is well-known that while the autocorrelation function of price returns decays rapidly, the autocorrelation function of the absolute values of price returns is power-law correlated in time (see [52] and extensive earlier work cited therein).

Consider, for example, the volatility. There are several possible definitions of this quantity, all of which seem to give the same scale invariant properties. But why care about volatility at all? On the cover of the May 15, 2000 issue of Forbes magazine is a large photograph of Henk Paulson, CEO of Goldman Sachs, and the headline quotation "Volatility is Our Friend." Why is this the case? Because it is known that volatility clusters, i.e., there are time correlations in this quantity. Our group has attempted to quantify these correlations, and found evidence of power law behavior [52, 56-58]. If we plot an economic earthquake such as Black Monday (19 October 1987) on which date most worldwide stock indices dropped 30-50\%, and then plot and compare the volatility (the absolute value of the fluctuations), we see a big peak in the volatility curve on Black Monday. But even prior to Black Monday the value of the volatility on our graph seems to be particularly unstable; there is some precursor to Black Monday evident in its behavior. One can imagine a computer program that would monitor volatility, not necessarily for the entire market but certainly for an individual stock, and the volatility calculation would need to be updated in real time.

There are correlations in the stock price change, but those correlations have a very short range - on the order of a few minutes - and they decay exponentially in time. Our group calculated the autocorrelation function of stock-price changes and plotted the logarithm of the function linearly in 


\section{H. E. Stanley et al.}

time; since the logarithm of $e^{-x}$ is $-x$, we get a straight line. In contrast, for the volatility we find that the autocorrelation function is linear on $\log -\log$ paper, meaning that the correlations in the volatility are power-law in nature. That, in turn, translates to mean they are much, much longer-range in time.

In order to quantify long-range power law volatility correlations, we developed a method of analyzing a non-stationary time series. The volatility of a financial market is non-stationary: there are days when the volatility is quiet and days when it is active. The statistical properties of a volatility time series are changing in time. The standard deviation of that time series is fluctuating wildly on every scale, which is the reason conventional methods are not effective. The method our group has been developing detrended fluctuation analysis (DFA) - gets rid of trends in the raw data [59-62]. We take a graph of the volatility expressed in absolute values (i.e., it is always positive) in which we see the peaks that indicate it is a very "noisy" or non-stationary time series, we integrate this time series, and we subtract the mean. This produces an up-and-down "landscape." We then look for correlations in this landscape. We do this by partitioning the landscape into "windowboxes" of a fixed size, e.g., 200 - does the regression fit to the fluctuations in that windowbox? We then calculate for each box the RMS fluctuation around the regression line. Finally, we average the RMS fluctuation for all 40,000 windowboxes of the entire series. With that many windowboxes, we get a very accurate measurement. We call the quantity $f$. We repeat the entire calculation for windowboxes one-half as big (size 100). Obviously, the smaller the windowbox, the less the fluctuation. This give us the circle for size 100. We repeat this a number of times. When that fluctuation is plotted as a function of windowbox size we find, contrary to what we might expect - that in almost all correlated signals the fluctuations increase as the square root of the windowbox size - the fluctuations instead increase more rapidly than that. That means there is some positive correlation in the signal. This analysis method produces results with very little noise. The data fall very close to the straight line, and the exponent can be obtained with a high degree of accuracy. All this allows us to analyze quantitatively the behavior of the volatility as a function of time and elucidate its correlations. This could be very useful information for people actually working in financial markets.

The distribution of volatility fluctuations has also been the object of extensive study. It was at one time believed by many that the volatility follows a log-normal distribution - i.e., the number of times the volatility has a certain value follows not a Gaussian but a $\log$-normal distribution, i.e., one has $e^{-(\ln x)^{2}}$ not $e^{-x^{2}}$. But until our group's work, no one had studied all the data: every trade [52]. Our doing it meant we could study relatively rare events, those occurring much less frequently than everyday events. What we find is that the log-normal part of the curve - the middle - though true for the middle, does not describe the tails. The huge volatilities in the tails are described by a different exponent $\mu$. We also see that volatility clusters - i.e., that volatility is correlated in time.

\section{Cross-Correlations Among Fluctuations of Different Stocks}

Another capability of such a software package could be the ability to determine how the fluctuations of one stock price correlate with those of another. This question of cross-correlation is one we have been studying [63-70].

To quantify cross-correlations,we draw a circle corresponding to the stock price $x$ and draw a second circle corresponding to the stock price $x$, say, 5 min later. If we make the difference in the radii proportional to $G$, the stock price change, then we can think of the market as thousands of circles, each growing and shrinking - a kind of pulsation that is a function of time. The key is that these correlations change in time. Car sales by Ford and GM may be anti-correlated during some time periods and positively correlated during others. 
The standard approach to this problem is to calculate, by brute force, a huge square matrix that has as many rows as there are companies in the database. Each element of the matrix is the correlation between the price change of company $i$ and the price change of company $j$, but to find a genuine correlation we have to be able to distinguish between correlations from coincidences. In order to do that we draw on something developed by Wigner in his work in nuclear physics - random matrix theory. Random matrix theory compares the matrix calculated by brute force from stock market data with a random matrix that also has 1000 rows and 1000 columns - but with every number generated randomly. Somewhere hidden in the huge matrix calculated by brute force from stock market data are the true correlations. To uncover them, we first diagonalize the matrix in order to determine its eigenvalues, and then make a histogram that gives the number of times each given eigenvalue is found. The histogram curve of a random matrix, unlike this one from real data, can be predicted exactly. For a random matrix there is never an eigenvalue $>2.0$. The histogram of the empirical stock price data, on the other hand, contains a significant number of eigenvalues $>2.0$. Some are as big as 5.0. These eigenvalues of necessity must correspond to genuine correlations.

The eigenvalue of a matrix has a corresponding eigenvector - a column matrix of 1000 elements each element of which is a different weight from each of the 1000 stocks. So we can look at the column vectors that correspond to these deviating, genuinely-correlated eigenvalues and ask: what kind of stocks entered into each of these eigenvectors? What we found, fortunately, has implications for portfolios. If we restart the graph at 2.0 - removing the distortions of the random values - and look at the 20 eigenvalues $>2.0$, we see that the stocks that make up most of the weights in the corresponding eigenvectors are almost entirely transportation stocks in the first case, almost entirely paper in the second, almost entirely pharmaceuticals in the third, and so on. In other words, the market automatically partitions itself into separate business sectors [69-71]. Thus a physicist who know nothing about the stock market can mathematically partition the economy into separate business sectors!

The sectors and the quantitative degree to which each constituent firm conforms to the sector can be monitored and updated as a function of time, e.g., every $15 \mathrm{~min}$. Firms that belong to the same business sector can be monitored in a kind of rainbow spectrum. The "good" firms sticking to the business sector are assigned to the "violet" end of the spectrum, and the "bad" firms deviating from the sector are assigned to the "red." When a firm first starts to move to the red end of the spectrum start to deviate, this alerts the trader to consider action.

\section{Equilibrium vs. Out-of-Equilibrium Market Phases}

Before concluding, we ask what sort of understanding could eventually develop if one takes seriously the power laws that appear to characterize finance fluctuations. It is tempting to imagine that there might be analogies between finance and known physical processes displaying similar scale-invariant fluctuations. One initially promising analogy was with turbulence: In turbulence, one adds energy at a large scale and this energy is dissipated at smaller and smaller scales in a scale-invariant fashion. Similarly, if external news is added at a large scale, then this news is dissipated by traders at smaller and smaller scales in a scale-invariant fashion. Despite some initial claims [72], these similarities are not borne out by quantitative analysis - although one finds non-Gaussian statistics, and intermittency, for both turbulence fluctuations and stock price fluctuations, the time evolution of the second moment and the shape of the probability density functions are different for turbulence and for stock market dynamics [73, 74].

More recent work pursues a rather different analogy, phase transitions in spin systems. It is not new to say that the set of all firm fluctuations is like a set of subunit fluctuations in a physics system such 
as a spin glass. Each fluctuation can be up or down, or any magnitude, and fluctuations interact with one another via interactions that are certainly long-range and of both signs. Further, the interactions change with time. A given subunit fluctuation is influenced (a) by other fluctuations (so the exchange interactions among spins is somewhat like the "herd effect"), and (b) by forces external to the system (so the external field is somewhat like "news" which plays a role in determining the sign and magnitude of fluctuations).

If this crude analogy were to hold even approximately, then a first step should perhaps be to seek to identify the analogs for the price fluctuation problem of field and temperature in the magnetic problem. Stock prices respond to demand, just as the magnetization of an interacting spin system responds to the magnetic field. Periods with large number of market participants buying the stock imply mainly positive changes in price, analogous to a magnetic field causing spins in a magnet to align. Recent work [75] quantifies the relations between price change and demand fluctuations, and finds results reminiscent of phase transitions in spin systems, where the divergent behavior of the response function at the critical point (zero magnetic field) leads to large fluctuations [9]. More precisely, buying and selling behavior in complex financial markets are driven by demand, which can be quantified by the imbalance in the number of shares transacted by buyers and sellers over a time interval $\triangle$.

If demand is the analog of magnetic field, then what is the analog of temperature? To answer this question, Plerou et al. [40, 41] analyze the probability distribution of demand, conditioned on its local noise intensity $\Sigma$, and find the surprising existence of a critical threshold $\Sigma_{c}$ separating two market phases. Their findings for the financial market problem are identical to what is known to occur in all phase transition phenomena, wherein the behavior of a system undergoes a qualitative change at a critical threshold $K_{c}$ of some control parameter $K$. Plerou et al interpret these two market phases as corresponding to two distinct conditions of the financial market: (a) The " $\Sigma<\Sigma_{c}$ market phase," where the distribution of demand is single peaked with the most probable value being zero, they interpret to be the market equilibrium phase, since the price of the stock is such that the probability of a transaction being buyer initiated is equal to the probability of a transaction being seller initiated, and (b) the " $\Sigma>\Sigma_{c}$ market phase," where the distribution of demand is bimodal, they interpret to be the out-of-equilibrium phase, since the price of the stock is such that there is an excess of either buyers or of sellers and there is a non-zero net demand for the stock.

It should be possible to design a software package that could be on every trader's desk allowing instant access to data on any firm in which time is partitioned into two different phases: equilibrium and out-of-equilibrium. Qualitatively and informally many people use those terms in reference to the stock market, but in this case we would be actually quantifying the extent to which the market is in or out of equilibrium. If we graph the price-change of a particular stock as a function of time for a sequence of 15-min intervals and use two different symbols for data points when the market is in equilibrium and for those for when it is out of equilibrium, we notice that in general a stock price is not changing when the market is in equilibrium and is changing when the market is out of equilibrium. This could be useful in that it could be an indicator of the relative stability of an individual stock. When the market is out of equilibrium, the probability that a stock price is going to change is higher than when the market is in equilibrium.

\section{Discussion}

Since the evidence for an analogy between stock price fluctuations and magnetization fluctuations near a critical point is backed up by quantitative analysis of finance data, it is legitimate to demand a 
theoretical reason for this analogy. To this end, we discuss briefly one possible theoretical understanding for the origin of scaling and universality in economic systems. As mentioned above, economic systems consist of interacting units just as critical point systems consist of interacting units. Two units are correlated in what might seem a hopelessly complex fashion - consider, e.g., two spins on a lattice, which are correlated regardless of how far apart they are. The correlation between two given spins on a finite lattice can be partitioned into the set of all possible topologically linear paths connecting these two spins - indeed this is the starting point of one of the solutions of the two-dimensional Ising model (see Appendix B of [9]). Since correlations decay exponentially along a one-dimensional path, the correlation between two spins would at first glance seem to decay exponentially. Now it is a mathematical fact that the total number of such paths grows exponentially with the distance between the two spins - to be very precise, the number of paths is given by a function which is a product of an exponential and a power law. The constant of the exponential decay depends on temperature while the constant for the exponential growth depends only on geometric properties of the system [9]. Hence by tuning temperature it is possible to achieve a threshold temperature where these two "warring exponentials" just balance each other, and a previously negligible power law factor that enters into the expression for the number of paths will dominate. Thus, power law scale invariance emerges as a result of canceling exponentials, and universality emerges from the fact that the interaction paths depend not on the interactions but rather on the connectivity. Similarly, in economics, two units are correlated through a myriad of different correlation paths; "everything depends on everything else" is the adage expressing the intuitive fact that when one firm changes, it influences other firms. A more careful discussion of this argument is presented, not for the economy but for the critical phenomena problem, in Ref. [10].

\section{Summary}

In summary, physicists are finding this emerging field fascinating. For a long time, physicists did relatively little in economics. A major reason for this is that, until recently, the amount of data routinely recorded concerning financial transactions was insufficient to be useful to physicists. That fact is no longer true. Now every trade is recorded, along with bid-ask quotes for every trade, and these data are made available.

Part of the reason for the invention of the neologism "econophysics" (in the tradition of the neologisms "biophysics," "astrophysics," "geophysics" ...) was to enable our physics students to persuade the departmental administrators that their dissertation research topics actually belonged in the physics department. The neologism seems to have caught on, and there are now several conferences each year with the word "econophysics" in the title.

Finally, a word of humility with respect to our esteemed economics colleagues is perhaps not inappropriate. Physicists may care passionately if there are analogies between physics systems they understand (like critical point phenomena) and economics systems they do not understand. But why should anyone else care? One reason is that scientific understanding of earthquakes moved ahead after it was recognized $^{\sim}[1,2]$ that extremely rare events - previously regarded as statistical outliers requiring for their interpretation a theory quite distinct from the theories that explain everyday shocks - in fact possess the identical statistical properties as everyday events; e.g., all earthquakes fall on the same straight line on an appropriate log-log plot. Since economic phenomena possess the analogous property, the challenge is to develop a coherent understanding of financial fluctuations that incorporates not only everyday fluctuations but also those extremely rare "financial earthquakes". 


\section{Acknowledgments}

We thank NSF for financial support and we thank our collaborators: L. A. N. Amaral, S. V. Buldyrev, D. Canning, P. Cizeau, S. Havlin, Y. Lee, Y. Liu, P. Maass, R. N. Mantegna, K. Matia, M. Meyer, B. Rosenow, M. A. Salinger, and M. H. R. Stanley, and most especially X. Gabaix, P. Gopikrishnan, and V. Plerou.

\section{References}

1. Gutenberg, B. and Richter, C. F., Seismicity of the Earth and Associated Phenomenon, 2nd Edn, Princeton University Press, Princeton, 1954

2. Turcotte, D. L., Fractals and Chaos in Geology and Geophysics, Cambridge University Press, Cambridge, 1992.

3. Rundle, J. B., Turcotte, D. L., and Klein, W., Reduction and Predictability of Natural Disasters, Addison-Wesley, Reading MA, 1996.

4. Gabaix, X., Gopikrishnan, P., Plerou, V., and Stanley, H. E., 'A theory of power-law distributions in financial Market Fluctuations', Nature 423, 2003, 267-270.

5. Gabaix, X., Gopikrishnan, P., Plerou, V., and Stanley, H. E., 'Institutional investors and stock market volatility', Quarterly Journal of Economics 121, 2006, 461-504.

6. Pareto, V., Cours d'Economie Politique, Lausanne, Paris, 1897.

7. Solomon, S. and Richmond, P., 'Stable power laws in variable economies; lotka-volterra implies pareto-zipf', European Physical Journal B 27, 2002, 257-261.

8. Mandelbrot, B. B., 'The variation of certain speculative prices', Journal of Business 36, 1963, 394-419.

9. Stanley, H. E., Introduction to Phase Transitions and Critical Phenomena, Oxford University Press, Oxford, 1971.

10. Stanley, H. E., 'Scaling, universality, and renormalization: Three pillars of modern critical phenomena', Review of Modern Physics 71, 1999, S358-S366.

11. Takayasu, H., ed., Empirical Science of Financial Fluctuations: The Advent of Econophysics, Springer, Berlin, 2002.

12. Mantegna, R. N. and Stanley, H. E., An Introduction to Econophysics: Correlations and Complexity in Finance, Cambridge University Press, Cambridge, 2000.

13. Bouchaud, J. P., 'Power laws in economics and finance: Some ideas from physics', Quantitative Finance 1, $2001,105-112$.

14. Bouchaud, J. P. and Potters, M., Theory of Financial Risk, Cambridge University Press, Cambridge, 2000.

15. Levy, H., Levy, M., and Solomon, S., Microscopic Simulation of Financial Markets, Academic Press, New York, 2000.

16. Roehner, B. M., Hidden Collective Factors in Speculative Trading, Springer, Berlin, 2001.

17. Roehner, B. M., Patterns of Speculation, Cambridge University Press, Cambridge, 2002.

18. Paul, W. and Baschnagel, A., Stochastic Processes from Physics to Finance, Springer, Berlin, 1999.

19. Voit, J., The Statistical Mechanics of Financial Markets, Springer, Berlin, 2001.

20. Axtell, R. L., 'Zipf distribution of US firm sizes', Science 293, 2001, 1818-1821.

21. Stanley, M. H. R., Buldyrev, S. V., Havlin, S., Mantegna, R., Salinger, M. A., and Stanley, H. E., 'Zipf plots and the size distribution of firms', Economics Letters 49, 453-457, 1996.

22. Stanley, M. H. R., Amaral, L. A. N., Buldyrev, S. V., Havlin, S., Leschhorn, H., Maass, P., Salinger, M. A., and Stanley, H. E., 'Scaling behavior in the growth of companies', Nature 379, 804-806, 1996.

23. Amaral, L. A. N., Buldyrev, S. V., Havlin, S., Leschhorn, H., Maass, P., Salinger, M. A., Stanley, H. E., and Stanley, M. H. R., 'Scaling behavior in economics: I. Empirical results for company growth', Journal of Physics I France 7, 621-633, 1997.

24. Buldyrev, S. V., Amaral, L. A. N., Havlin, S., Leschhorn, H., Maass, P., Salinger, M. A., Stanley, H. E., and Stanley, M. H. R., 'Scaling Behavior in Economics: II. Modeling of Company Growth', Journal of Physics I France 7, 635-650, 1997.

25. Takayasu, H. and Okuyama, K., 'Country dependence on company size distributions and a numerical model based on competition and cooperation', Fractals 6, 67-79, 1998.

26. Plerou, V., Amaral, L. A. N., Gopikrishnan, P., Meyer, M., and Stanley, H. E., 'Similarities between the growth dynamics of university research and of competitive economic activities', Nature 400, 433-437, 1999.

27. Canning, D., Amaral, L. A. N., Lee, Y., Meyer, M., and Stanley, H. E., 'A power law for scaling the volatility of GDP growth rates with country size', Econ. Lett. 60, 335-341, 1998.

28. Lee, Y., Amaral, L. A. N., Canning, D., Meyer, M., and Stanley, H. E., 'Universal features in the growth dynamics of complex organizations', Physical Review Letters 81, 3275-3278, 1998.

29. Keitt, T. and Stanley, H. E., 'Scaling in the dynamics of North American breeding-bird populations', Nature 393, $257,1998$.

30. Amaral, L. A. N., Buldyrev, S. V., Havlin, S., Salinger, M. A., and Stanley, H. E., 'Power law scaling for a system of interacting units with complex internal structure', Physical Review Letters 80, 1385-1388, 1998. 
31. Sutton, J., 'The variance of firm growth rates: The scaling puzzle', Physica A 312, 2002, 577.

32. Cecconi, F., Marsili, M., Banavar, J. R., and Maritan, A., 'Diffusion, peer pressure, and tailed distributions', Physical Review Letters 89, 2002, 088102.

33. Wyart, M., and Bouchaud, J.-P., 'Statistical models for company growth', cond-mat/0210479, October 2002.

34. Lux, T., 'The stable paretian hypothesis and the frequency of large returns: An examination of major german stocks', Applied Financial Economics 6, 463-475, 1996.

35. Gopikrishnan, P., Meyer, M., Amaral, L. A. N., and Stanley, H. E., 'Inverse cubic law for the distribution of stock price variations', European Physical Journal B 3, 139-140, 1998.

36. Plerou, V., Gopikrishnan, P., Amaral, L. A. N., Meyer, M., and Stanley, H. E., 'Scaling of the distribution of price fluctuations of individual companies', Physical Review E 60, 6519-6529, 1999.

37. Gopikrishnan, P., Plerou, V., Amaral, L. A. N., Meyer, M., and Stanley, H. E., 'Scaling of the distributions of fluctuations of financial market indices', Physical Review E 60, 5305-5316, 1999.

38. Matia, K., Amaral, L. A. N., Goodwin, S., and Stanley, H. E., 'Non-Lévy distribution of commodity price fluctuations', Physical Review E: Rapid Communications 66, 045103, 2002. cond-mat/0202028.

39. Matia, K., Ashkenazy, Y., and Stanley, H. E., 'Multifractal properties of price fluctuations of stocks and commodities', Europhysics Letters 61, 422-428, 2003.

40. Plerou, V., Gopikrishnan, P., and Stanley, H. E., 'Two-phase behaviour of financial markets', Nature 421, 130, 2003. condmat/0111349.

41. Plerou, V., Gopikrishnan, P., and Stanley, H. E., 'Symmetry breaking in stock demand', Physical Review E (submitted) cond-mat/0111349.

42. Mantegna, R. N. and Stanley, H. E., 'Stochastic process with ultraslow convergence to a gaussian: The truncated 1 évy flight', Physical Review Letters 73, 2946-2949, 1994.

43. Mantegna, R. N. and Stanley, H. E.,'Scaling behavior in the dynamics of an economic index,' Nature 376, 46-49, 1995.

44. Podobnik, B., Ivanov, P. Ch., Lee, Y., Chessa, A., and Stanley, H. E., 'Systems with correlations in the variance: Generating power law tails in probability distributions', Europhysics Letters 50, 2000 711-717.

45. Mantegna, R. N. and Stanley, H. E., 'Ultra-slow convergence to a gaussian: The truncated lévy flight', in Lévy Flights and Related Topics in Physics [Proceedings of 1994 International Conference on Lévy Flights], M. F. Shlesinger, G. M. Zaslavsky, and U. Frisch (eds.), Springer, Berlin, 1995, pp. 300-312.

46. Mantegna, R. N. and Stanley, H. E., 'Modeling of financial data: Comparison of the truncated lévy flight and the ARCH(1) and GARCH(1,1) processes', [Proceedings of Int' l IUPAP Conference on Statistical Physics, Taipei], Physica A 254, 77-84, 1998.

47. Podobnik, B., Ivanov, P. Ch., Lee, Y., and Stanley, H. E., 'Scale-invariant Truncated Lévy Process', Europhysics Letters 52, 491-497, 2000

48. Ivanov, P. Ch., Podobnik, B., Lee, Y., and Stanley, H. E., 'Truncated Lévy Process with Scale-Invariant Behavior', [Proceedings of NATO Advanced Research Workshop on Application of Physics in Economic Modeling, Prague], Physica A 299, 154-160, 2001.

49. Skjeltorp, J. A., 'Scaling in the Norwegian stock market', Physica 283, 486-528, 2001.

50. LeBaron, B., 'Stochastic volatility as a simple generator of financial power laws and long memory', Quantitative Finance $\mathbf{2}$, $621-631,2001$.

51. Stanley, H. E. and Plerou, V., 'Scaling and universality in economics: Empirical results and theoretical interpretation', Quantitative Finance 1, 563-567, 2001.

52. Liu, Y., Gopikrishnan, P., Cizeau, P., Meyer, M., Peng, C. K., and Stanley, H. E., 'The statistical properties of the volatility of price fluctuations', Physical Review E 60, 1390-1400, 1999.

53. Plerou, V., Gopikrishnan, P., Amaral, L. A. N., Gabaix, X., and Stanley, H. E., 'Diffusion and economic fluctuations', Physical Review E (Rapid Communications) 62, 3023-3026, 2000.

54. Gopikrishnan, P., Plerou, V., Gabaix, X., and Stanley, H. E., 'Statistical properties of share volume traded in financial markets', Physical Review E (Rapid Communications) 62, 4493-4496.

55. Plerou, V., Gopikrishnan, P., Gabaix, X., Amaral, L. A. N., and Stanley, H. E., 'Price fluctuations, market activity, and trading volume', [Proceedings of 2000 Santa Fe Econophysics Conference], Quantitative Finance 1, 262-269, 2001.

56. Liu, Y., Cizeau, P., Meyer, M., Peng, C.-K., and Stanley, H. E., 'Quantification of correlations in economic time series', Physica A 245, 437-440, 1997.

57. Cizeau, P., Liu, Y., Meyer, M., Peng, C.-K., and Stanley, H. E., 'Volatility distribution in the S\&P500 Stock Index', Physica A 245, 441-445, 1997.

58. Podobnik, B., Matia, K., Chessa, A., Ivanov, P. Ch., Lee, Y., and Stanley, H. E., 'Time evolution of stochastic processes with correlations in the variance: Stability in power-law tails of distributions', Physica A 300, 300-309, 2001.

59. Peng, C.-K., Buldyrev, S. V., Havlin, S., Simons, M., Stanley, H. E., and Goldberger, A. L., 'On the mosaic organization of DNA sequences', Physical Review E 49, 1691-1695, 1994.

60. Hu, K., Chen, Z., Ivanov, P. Ch., Carpena, P., and Stanley, H. E., 'Effect of trends on detrended fluctuation analysis', Physical Review E 64, 011114-1, 2001. physics/0103018. 
340 H. E. Stanley et al.

61. Chen, Z., Ivanov, P. Ch., Hu, K., and Stanley, H. E., 'Effect of nonstationarities on detrended fluctuation analysis', Physical Review E 65, 041107-1, 2002. physics/0111103.

62. Kantelhardt, J. W., Zschiegner, S., Koscielny-Bunde, E., Havlin, S., Bunde, A., and Stanley, H. E., 'Multifractal detrended fluctuation analysis of nonstationary time series', Physica A 316, 87, 2002.

63. Guhr, T., Müller-Groeling, A., and Weidenmüller, H. A., 'Random-matrix theories in quantum physics: Common concepts', Physics Reports 299, 1998, 189-425.

64. Mehta, M. L. and Dyson, F. J., 'Statistical theory of the energy levels of complex systems. V', Journal of Mathematical Physics 4, 713-719, 1963.

65. Dyson, F. J., 'The distribution of singular values of certain random matrices', Revista Mexicana de Física 20, $231,1971$.

66. Sengupta, A. M. and Mitra, P. P., 'Distributions of singular values for some random matrices', Physical Review E 60, 3389-3392, 1999

67. Laloux, L., Cizeau, P., Bouchaud, J.-P., and Potters, M., 'Noise dressing of financial correlation matrices', Physical Review Letters 83, 1469-1482, 1999.

68. Plerou, V., Gopikrishnan, P., Rosenow, B., Amaral, L. A. N., and Stanley, H. E., 'Universal and nonuniversal properties of financial cross-correlation matrices', Physical Review Letters 83, 1471-1475, 1999.

69. Gopikrishnan, P., Rosenow, B., Plerou, V., and Stanley, H. E., 'Quantifying and interpreting collective behavior in financial markets', Physical Review E: Rapid Communications 64, 035106, 2001.

70. Plerou, V., Gopikrishnan, P., Rosenow, B., Amaral, L. A. N., Guhr, T., and Stanley, H. E. 'A random matrix approach to financial cross-correlations'. Physical Review E 65, 066126, 2002. cond-mat/0108023.

71. Rosenow, B., Plerou, V., Gopikrishnan, P., and Stanley, H. E., 'Portfolio optimization and the random magnet problem', Europhysics Letters 59, 500-506, 2002. cond-mat/0111537.

72. Ghashgaie, S., Breymann, W., Peinke, J., Talkner, P., and Dodge, Y., 'Turbulent cascades in foreign exchange markets', Nature 381, 767-770, 1996.

73. Mantegna, R. N. and Stanley, H. E., 'Turbulence and exchange markets', Nature 383, 587-588, 1996.

74. Mantegna R. N. and Stanley, H. E., 'Stock market dynamics and turbulence: Parallel analysis of fluctuation phenomena', [Proceedings of International Conference on Pattern Formation in Fluids and Materials], Physica A 239, $255-266,1997$.

75. Plerou, V., Gopikrishnan, P., Gabaix, X., and Stanley, H. E., 'Quantifying stock price response to demand fluctuations', Physical Review E 66, 027104, 2002. cond-mat/0106657. 